\title{
Infrared Small Target Detection Method Combined with Bilateral Filter and Local Entropy
}

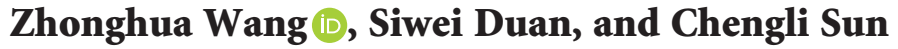 \\ School of Information Engineering, Nanchang Hangkong University, Nanchang 330063, China \\ Correspondence should be addressed to Zhonghua Wang; wangzhonghuawzh@126.com
}

Received 31 December 2020; Revised 26 January 2021; Accepted 2 February 2021; Published 13 February 2021

Academic Editor: Nanrun Zhou

Copyright (c) 2021 Zhonghua Wang et al. This is an open access article distributed under the Creative Commons Attribution License, which permits unrestricted use, distribution, and reproduction in any medium, provided the original work is properly cited.

\begin{abstract}
According to the larger false alarm rate resulted from fluctuant clutter, a novel method combined with bilateral filter and local entropy is proposed for infrared small target detection in this paper. Firstly, the original image is respectively processed by bilateral filter and local entropy, and then the two processed images are fused by point product to generate the background suppression map. Secondly, the guided filter is used to further suppress the background and enhance the small target in the map. Thirdly, the small target is detected by the adaptive threshold in the filtered map. The theoretical analyses and experimental results show that the proposed method not only effectively suppresses the clutter background, depending on the edge preserving and denoising characteristics of bilateral filtering, but also effectively highlights the small target, relying on the sensitivity of local entropy to the abrupt gray region. Compared with other methods, it is demonstrated that the proposed method owns lower false alarm rate and higher detection rate.
\end{abstract}

\section{Introduction}

For the advantages of antijamming, concealing, and all weather, the infrared technology is widely used in the military domain, such as target tracking and detection and antimissile. However, due to the infrared remote imaging, the target occupies only a few pixels in the infrared image so that it has no shape, size, and texture information [1-3]. Moreover, because of the strong clutter jamming, the target is mostly immersed in the background.

Consequently, how to accurately detect the small target has become one of the research hotspots and has attracted attention of scholars around the world.

In images, the gray values of noise and edge have step changes, and in some cases, they are not easy to distinguish. Therefore, how to effectively remove noise and retain more detailed features is the key to image denoising. Bilateral filtering algorithm, which compromises the spatial proximity and gray similarity, has been widely used because of its good filtering effect and edge preserving characteristics [4]. In the smoothing region of image, the spatial Euclidean distance plays a decisive role, while at the edge of image, the gray similarity, plays a decisive role. For the small target region, it belongs to the high frequency component of the image [5]. To enhance the discontinuity between the target area and the surrounding area, a method of combining gray difference and bilateral filtering [6] was proposed by Zhu et al. However, the dim target region affects the background prediction. Yuan et al. proposed an improved bilateral filtering to detect the multiscale moving target [7], but the residual background textures affect the quality of the background suppression map.

Entropy is a measure of the uncertainty of the probability of the occurrence of events in information theory. For the background image, because its texture features are determined, its entropy should be determined. However, when the target appears in the image, the texture features of the image are destroyed, and the image entropy must change [8]. Sun et al. proposed an improved weighted local entropy method, which reflects the background characteristic difference [9], but the strong clutter interference is not well solved. 
For the residual image of bilateral filtering, it retains not only the small target but also the background texture. However, for the local entropy image, it reserves the small target and the strong clutter background. The presence of the texture and clutter interferes with the accuracy of small target detection [10]. To improve the accuracy of target detection, a small infrared target detection algorithm based on bilateral filtering and local entropy is proposed in this paper.

The algorithm respectively uses bilateral filtering to predict the image background and adopts local entropy to generate the entropy image, and then the dot product of the residual image and local entropy image is calculated. The fused image indicates that the background clutter and texture are greatly suppressed while the targets are effectively retained. Subsequently, the guided filtering is introduced to further enhance the target, which is helpful for small target segmentation. The experimental results indicate that the proposed algorithm can effectively suppress the background component and detect the small target. The rest of this paper is organized as follows. Section 2 introduces the principle of local entropy. The infrared small target detection method combined with bilateral filtering and local entropy is proposed in Section 3. Section 4 shows the experimental analysis and comparison. The conclusion is depicted in Section 5.

\section{Local Entropy Principle}

Entropy was first proposed by German physicist Rudolf Clausius in 1865 and applied to thermodynamics. Then, in 1948, American engineer Shannon first introduced it into information theory. Entropy in information theory means the average amount of information contained in each message received [11].

If there are multiple events $S=\left\{E_{1}, E_{2}, \ldots, E_{n}\right\}$ in a system $S$ and the probability distribution of each event $P=\left\{p_{1}, p_{2}, \ldots, p_{n}\right\}$, the information of each event is defined by the following formula:

$$
H_{e}=-\log _{2} p_{i} \quad(i=1, \ldots, n) .
$$

Then, the average amount of information of the whole system is as follows:

$$
H_{s}=\sum_{i=1}^{n} p_{i} H_{e}=-\sum_{i=1}^{n} p_{i} \log _{2} p_{i} .
$$

Image entropy is generally considered as a measure of uncertainty of image gray spatial distribution $[12,13]$. For an $m \times n$ image $f$, according to Shannon entropy theory, its image entropy can be defined as follows:

$$
H_{f}=-\sum_{i=1}^{n} \sum_{j=1}^{n} p_{i j} \log _{2} p_{i j}
$$

where $p_{i j}=\left(f(i, j) / \sum_{i=1}^{n} \sum_{j=1}^{n} f(i, j)\right)(f(i, j)$ represents the gray value of $(i, j)$ coordinates of the image $f)$.

If $m \times n$ is the image local window, formula (3) represents the image local entropy. The local entropy reflects the discrete degree of gray level in the window. Since the gray distribution of the slow change area is relatively uniform, the local entropy of the region is large, while according to the gray fluctuation region, the local entropy of the region is small. On the basis of the change of local entropy, the small target can be detected.

\section{Improved Detection Method for Infrared Small Target (IDMST)}

In this paper, an improved method is proposed for infrared small target detection. The IDMST is divided into three steps. Firstly, the original image is respectively processed by the bilateral filtering and local entropy, and then the correspondingly generated residual image and local entropy image are fused by point product to generate the background suppression map. Secondly, the guided filtering is used to further enhance the small target and suppress the leakage background in the map. Thirdly, the small target is detected by the adaptive threshold in the filtered map.

The implementation process of the IDMST is demonstrated in Figure 1.

3.1. Bilateral Filter Preprocessing. Bilateral filter is a nonlinear image filtering method, which considers the spatial proximity and gray value similarity of an image to implement the image background prediction. Then, the residual image $J$ is obtained by subtracting the predicted background image from the original image $I$, and its definition is as follows:

$$
\begin{aligned}
J(x, y)= & I(x, y)-\sum_{\left(x_{i}, y_{i}\right) \in \Omega} I\left(x_{i}, y_{i}\right) * W\left(x_{i}, y_{i}\right), \\
W\left(x_{i}, y_{i}\right)= & \frac{1}{C} \sum_{\left(x_{i}, y_{i}\right) \in \Omega} G_{\delta_{d}}\left(\left\|\left(x_{i}, y_{i}\right)-(x, y)\right\|\right) \\
& * G_{\delta_{r}}\left(\left\|I\left(x_{i}, y_{i}\right)-I(x, y)\right\|\right), \\
G_{\delta_{d}}\left(\left\|\left(x_{i}, y_{i}\right)-(x, y)\right\|\right)= & \exp \left(-\frac{\left\|\left(x_{i}, y_{i}\right)-(x, y)\right\|}{2 \delta_{d}^{2}}\right), \\
G_{\delta_{r}}\left(\left\|I\left(x_{i}, y_{i}\right)-I(x, y)\right\|\right)= & \exp \left(-\frac{\left\|I\left(x_{i}, y_{i}\right)-I(x, y)\right\|}{2 \delta_{r}^{2}}\right),
\end{aligned}
$$

where $I(x, y)$ represents the pixel gray value at the $(x, y)$ coordinates of the original image $I, W\left(x_{i}, y_{i}\right)$ is the masked coefficient of the bilateral filter, $\Omega$ is the definition domain of the neighborhood of the central point $(x, y)$, and $G_{\delta_{d}}$ and $G_{\delta_{r}}$ represent the Gaussian kernel functions of the spatial proximity and the gray value similarity, respectively. 


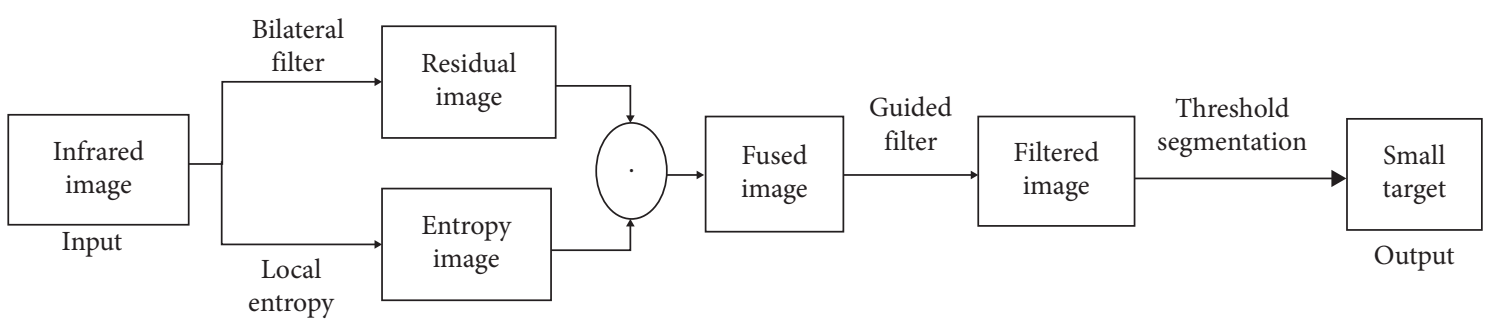

FIGURE 1: IDMST procedure.

According to reference [8], the performance of the guided filtering is better when $\delta_{d}$ is taken as $0.1-0.6$ and $\delta_{r}$ is in the range from 2 to 3 . Figure 2 shows the results of bilateral filter preprocessing.

As seen in Figure 2, the smoothing background or the strong clutter background is suppressed while the small target or the texture background is retained in the residual image; in addition, the small target is enhanced compared with its neighborhood space.

3.2. Local Entropy Computation. According to formula (3), the local entropy map $H_{f}$ is calculated. For the strong change region in the original image, its entropy is relatively smaller, while for the smoothing region, its entropy is larger [14]. Therefore, the small target and the strong clutter in the original image will be suppressed.

Since the contrast between the target and its neighborhood background is low, the entropy value is remapped to the $0-255$ range, and the conversion formula is shown in the following equation:

$$
H(i, j)=255-\frac{\left[H_{f}(i, j)-H_{f \min }\right] \times 255}{H_{f \max }-H_{f \min }},
$$

where $H_{f \text { min }}$ is the maximum and $H_{f \max }$ is the minimum in the local entropy map $H_{f}$ and $H(i, j)$ is the remapped value with $(i, j)$ coordinators.

Since formula (5) owns the feature of image reversal, the small target or the strong clutter is enhanced while the smoothing background is restrained.

In this paper, the generated residual image and the local entropy map are fused by dot product to obtain the background suppression image $S$, where most of the flat background and the fluctuant background are restrained and the small target is clearly enhanced. The definition of the background suppression image $S$ is as follows:

$$
S(i, j)=J(i, j) . * H(i, j) .
$$

3.3. Guided Filtering. Since the background suppression image $S$ still retains the leaked background, to make the target more prominent, the guided filtering is used to process the fused image to restrain the leaked background and enhance the target component, which is conducive to the subsequent image segmentation $[15,16]$.

Similar to bilateral filtering, the guided filtering is a nonlinear filtering method, but it has unique advantages, which have lower mathematical calculation and less image distortion.

The guided filtering satisfies the following linear model:

$$
q_{i}=a_{k} I_{i}+b_{k}, \quad\left(i \in \omega_{k}\right),
$$

where $I$ is the guided image, $q$ is the output image, $i$ is the pixel index, $\omega_{k}$ is a window centered on the $k$ th pixel of the guided image, and $a_{k}$ and $b_{k}$ are the linear coefficients corresponding to the window.

To acquire $a_{k}$ and $b_{k}$, the following objective function is defined to solve the optimization problem of the minimum difference between the output image $q$ and the input image $p$,

$$
E\left(a_{k}, b_{k}\right)=\sum_{i \in \omega_{k}}\left(\left(a_{k} I_{i}+b_{k}-p_{i}\right)^{2}+\varepsilon a_{k}^{2}\right) .
$$

In expression (8), $a_{k}$ and $b_{k}$ can be obtained by the least square method:

$$
\begin{aligned}
& a_{k}=\frac{(1 /|\omega|) \sum_{i \in \omega_{k}} I_{i} p_{i}-\mu_{k} \bar{p}_{k}}{\sigma_{k}^{2}+\varepsilon}, \\
& b_{k}=\bar{p}_{k}-a_{k} \mu_{k},
\end{aligned}
$$

where $\mu_{k}$ and $\sigma_{k}$ are the mean and variance of the guide image in the window, respectively, $\bar{p}_{k}$ is the mean of the input image in the window, and $|\omega|$ is the total number of pixels in the window.

Provided that $a_{k}$ and $b_{k}$ are solved, the output image $q$ can be generated by formula (7). Then, the image $q$ is segmented by the adaptive threshold to obtain the small target. The threshold $T h$ is set as follows:

$$
\mathrm{Th}=\mu_{q}+k \times \sigma_{q},
$$

where $\mu_{q}$ and $\sigma_{q}$ are the mean and variance of the output image, respectively, and $k$ has influence to the target detection result. If $k$ is too large, the real target is missed; otherwise, if $k$ is too small, the false target is misdetected. To balance the detection rate and false alarm rate, $k$ is set between 1 and 3 in the proposed method. 


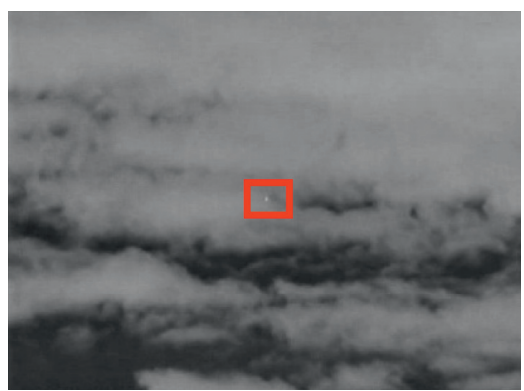

(a)

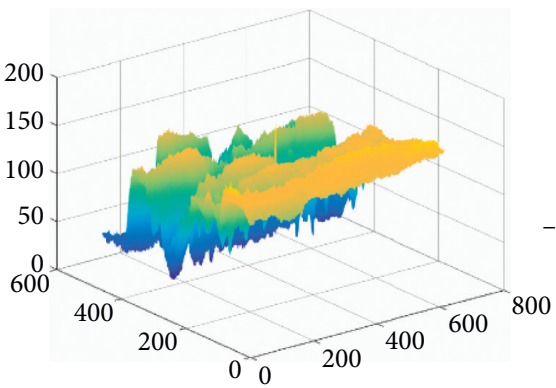

(d)

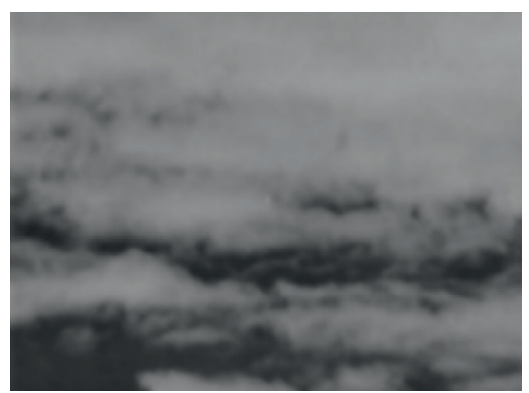

(b)



(c)

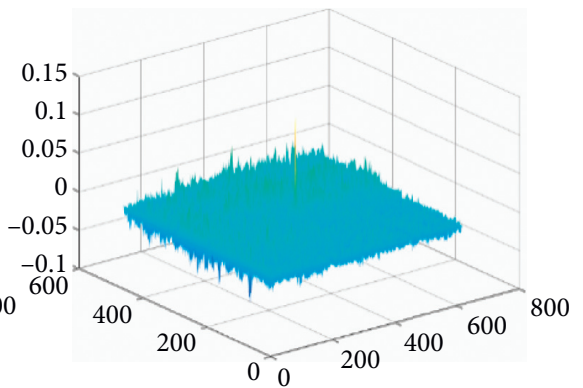

(e)

Figure 2: Bilateral filter preprocessing. (a) Original image. (b) Background prediction. (c) Residual image. (d) Three-dimensional map of original image. (e) Three-dimensional map of residual image.

\section{Simulation Results and Discussions}

In this paper, the IDMST is compared with bilateral filtering method [17], local entropy method [18], and max-mean method [19] to test the performances of background suppression and target detection. The parameter setting of each method is shown in Table 1. The first group of experiments is to evaluate the background suppression capability. The second group of experiments verifies the target detection performance. All experimental programs are compiled by MATLAB r2016a software and run on a computer with 2.7 GHz Intel Core i7 and 16 GB memory.

4.1. Comparison of Background Suppression Performance. In this experiment, three groups of infrared images with different backgrounds in Figure 3 are selected for the comparative test. In Figure 3(a), the background of the original image 1 is a large area of thick clouds, and its change is relatively slow. In Figure 3(b), the background of the original image 2 is the structured cloud edge, and the target is located on the sharp clutter edge. The background of the original image 3 in Figure 3(c) contains a lot of strong cloud clutters, and the target is submerged in the cloud. The common ground of the three groups of images is the scenes of flying small targets in the sky, and the background is more complex. Small target areas in Figure 3 are marked with red boxes.

As shown in Figure 3, there are the background suppression maps of infrared images of different scenes before and after processing by different algorithms. In Figure 3(d), the bilateral filtering method has good background suppression performance for thick cloud, but in Figures 3(e) and 3(f), the background cannot be effectively filtered out for the cloud region with complex texture. In Figures 3(g)-3(i), the local entropy method can effectively detect the gray mutation area, but cannot distinguish the mutations of cloud edge and target area. In Figures 3(j)$3(1)$, the target is not prominent and is distorted. Compared with other methods, it is shown in Figures $3(\mathrm{~m})$ and 3 (o) that our method IDMST can fully suppress the background clutter interference and highlight the small target. Although the IDMST cannot completely filter out the clutter in Figure 3(n), it has better background suppression effect than other methods.

As shown in Figure 4, there are three-dimensional response maps of infrared images of different scenes before and after processing by different algorithms. The three-dimensional response map shows the changes of image details more easily, which is conducive to subsequent analysis and comparison. From the three-dimensional maps processed by the IDMST, it is seen in Figures $4(\mathrm{~m})-4(\mathrm{o})$ that different backgrounds are mostly suppressed and the targets are very prominent around their neighborhood.

Therefore, the improved small target detection algorithm based on bilateral filtering and local entropy not only has good clutter background suppression ability but also ensures that the target structure is not damaged by background restraint and is highlighted.

To evaluate the background suppression performance of each algorithm more clearly, the local signal-to-clutter ratio (LSCR), the signal-to-clutter ratio gain coefficient (SCRG), 
TABLe 1: Parameter setting of each method.

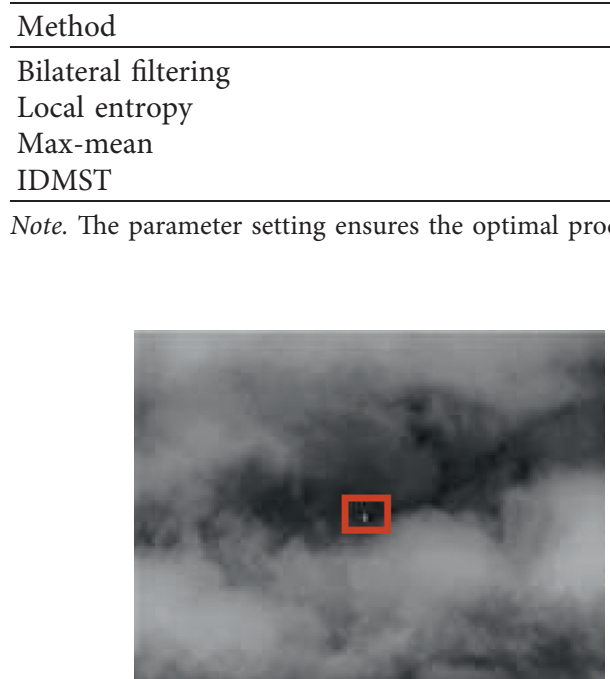

(a)

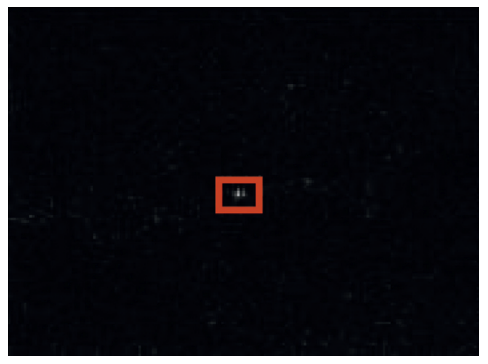

(d)

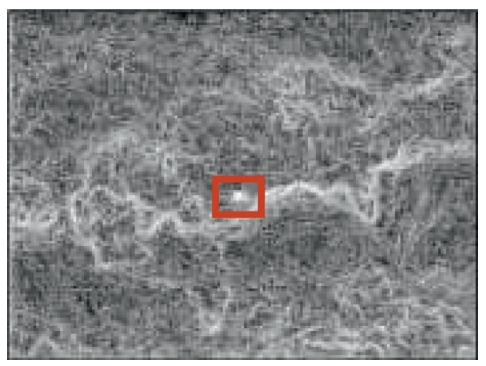

(g)

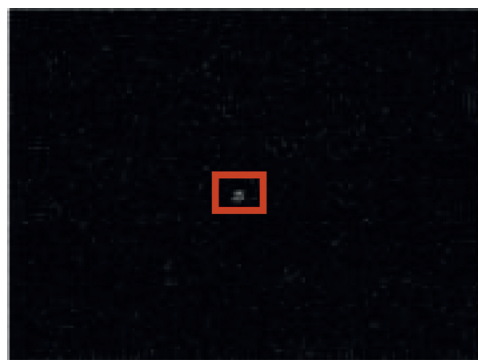

(j)

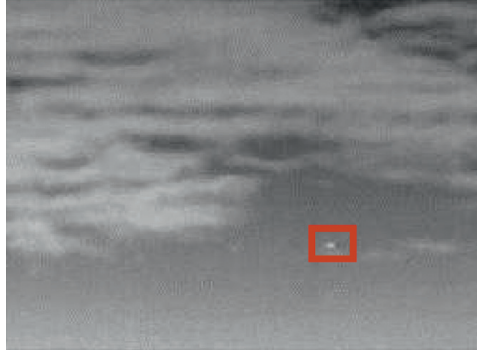

(b)

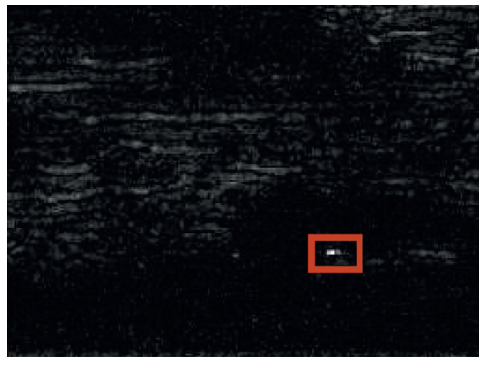

(e)

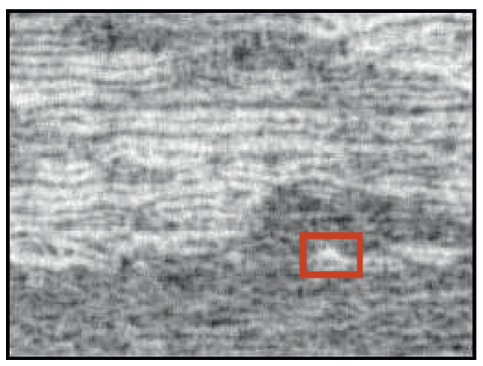

(h)

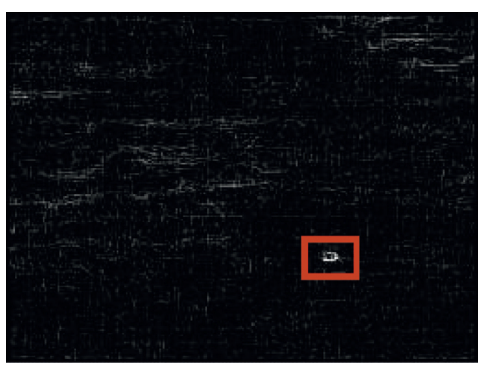

(k)

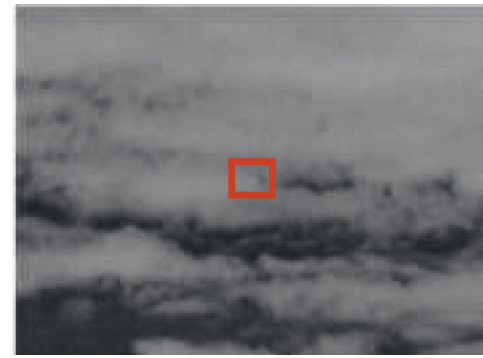

(c)

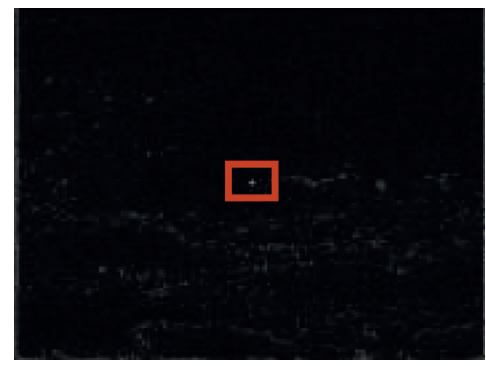

(f)

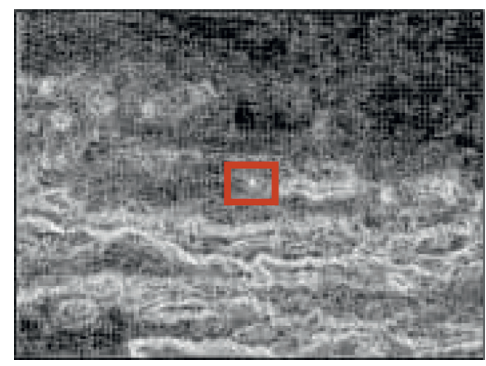

(i)

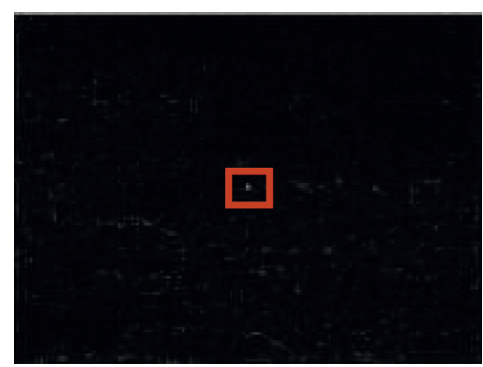

(1)

Figure 3: Continued. 


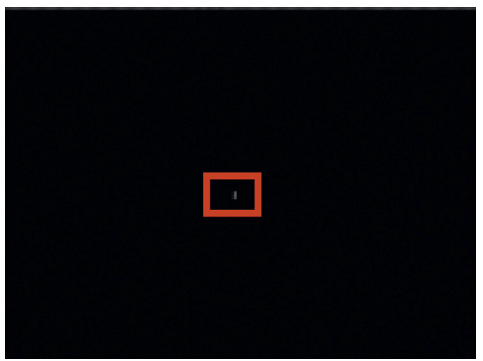

$(\mathrm{m})$

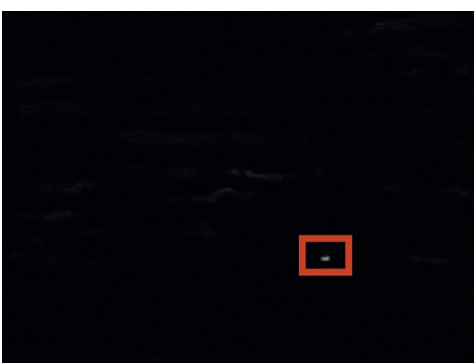

(n)

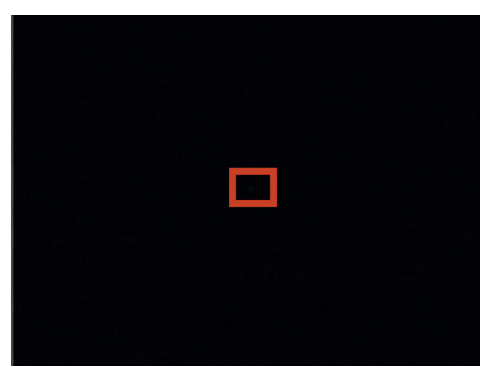

(o)

Figure 3: Background suppression maps of different methods. (a) Infrared original image 1. (b) Infrared original image 2. (c) Infrared original image 3. (d) Bilateral filtering 1. (e) Bilateral filtering 2. (f) Bilateral filtering 3. (g) Local entropy 1. (h) Local entropy 2. (i) Local entropy 3. (j) Max-mean 1. (k) Max-mean 2. (l) Max-mean 3. (m) IDMST 1. (n) IDMST 2. (o) IDMST 3.



(a)

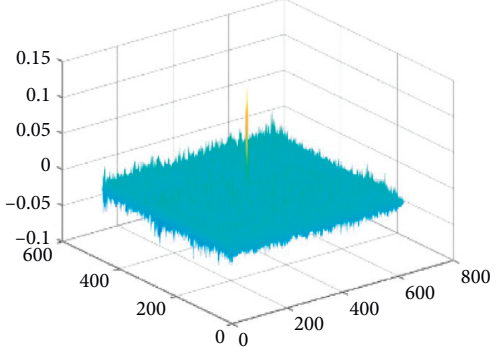

(d)

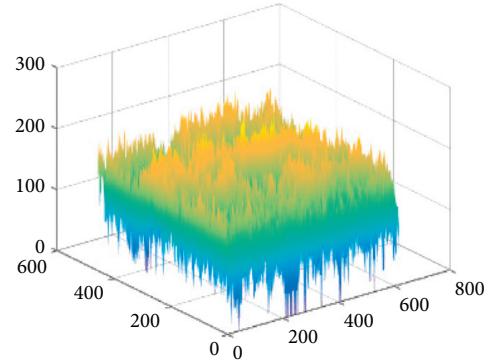

(g)

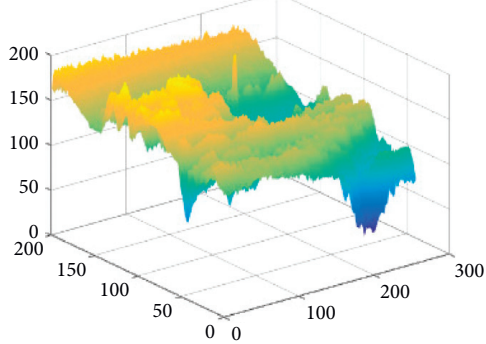

(b)

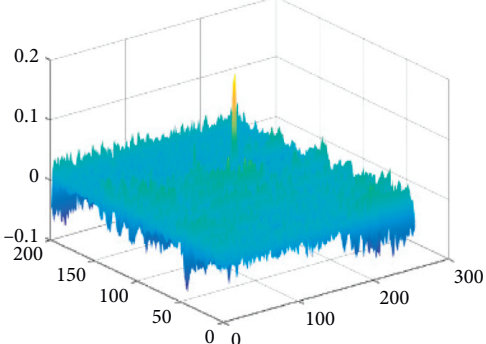

(e)

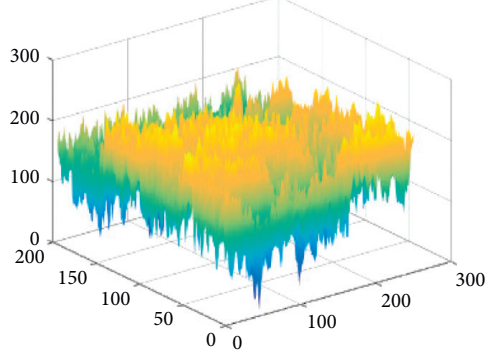

(h)

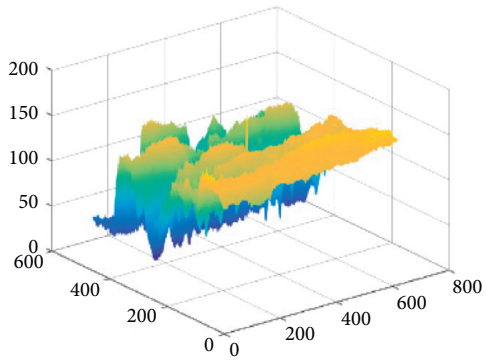

(c)

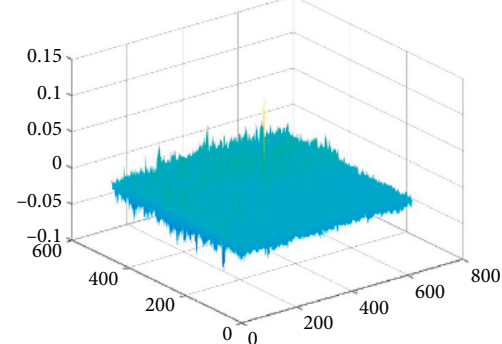

(f)

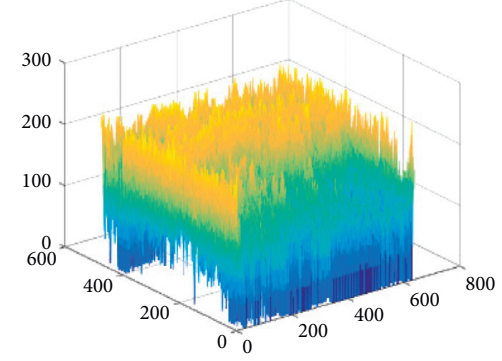

(i)

FIgURE 4: Continued. 


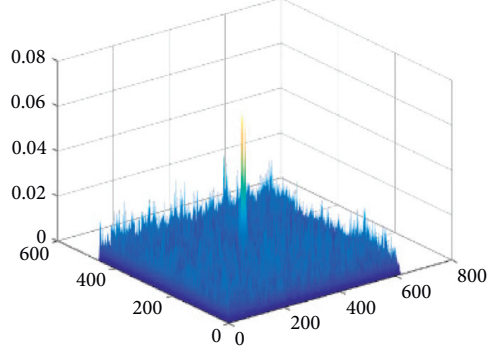

(j)

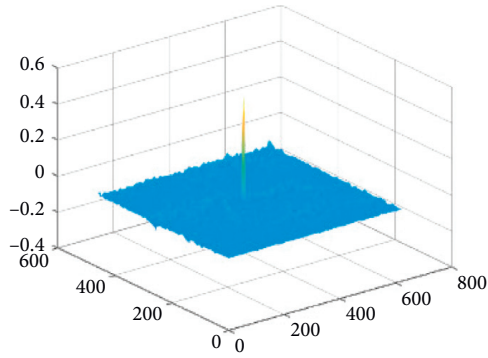

(m)

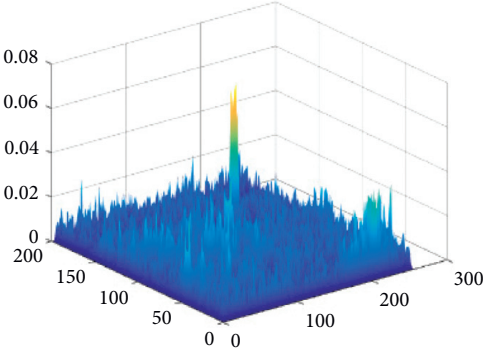

(k)

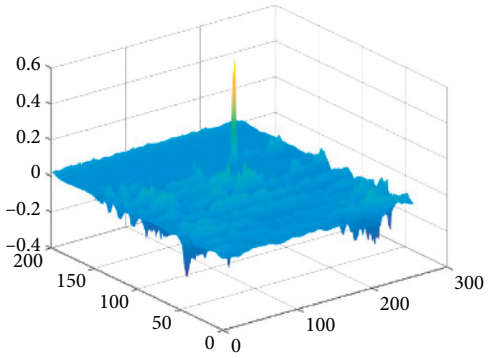

(n)

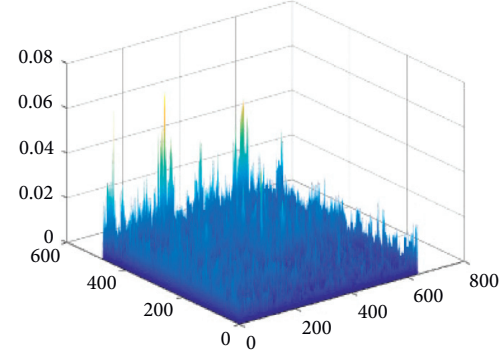

(l)

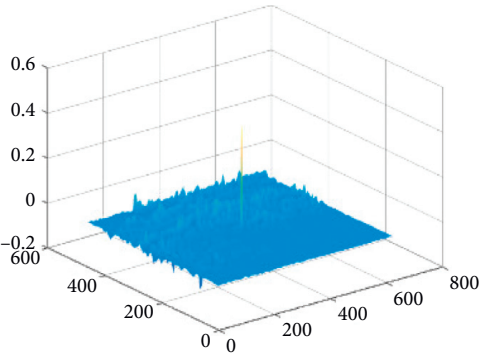

(o)

FIGURE 4: 3D response maps of different methods. (a) Infrared original image 1. (b) Infrared original image 2. (c) Infrared original image 3. (d) Bilateral filter 1. (e) Bilateral filter 2. (f) Bilateral filter 3. (g) Local entropy 1. (h) Local entropy 2. (i) Local entropy 3. (j) Max-mean 1. (k) Max-mean 3. (l) Max-mean 3. (m) IDMST 1. (n) IDMST 2. (o) IDMST 3.

and the background suppression factor (BSF) are used to quantitatively compare. Their definitions are as follows, respectively $[20,21]$ :

$$
\begin{aligned}
\operatorname{LSCR} & =\frac{\left|I_{\text {max }}-\mu_{b}\right|}{\sigma_{b}}, \\
\operatorname{SCRG} & =\frac{\mathrm{SCR}_{\text {out }}}{\mathrm{SCR}_{\text {in }}}, \\
\mathrm{BSF} & =\frac{\sigma_{\text {in }}}{\sigma_{\text {out }}},
\end{aligned}
$$

where $I_{\max }$ represents the maximum gray value of the pixel in the region where the small target is located, $\mu_{b}$ and $\sigma_{b}$ are the mean and standard deviation of the gray levels of the pixels in the target neighborhood, respectively, $\mathrm{SCR}_{\text {in }}$ and $\mathrm{SCR}_{\text {out }}$ are the signal-to-clutter ratios of the original image and the processed image, respectively, and $\sigma_{\text {in }}$ and $\sigma_{\text {out }}$ are the standard deviations of the original image and the processed image, respectively.

The larger the LSCR is, the more prominent the target is and the easier it is to detect. The larger the SCRG, the stronger the ability of suppressing background clutter and enhancing target. The larger the BSF, the better the background suppression effect will be. The comparison results of the three indicators are shown in Tables 2-4.

As seen in Tables $2-4$, it can be concluded that, for different background images, the performances of background suppression and target enhancement of our algorithm IDMST are the best among the five methods.
4.2. Detection Performance Comparison. In this experiment, three groups of infrared image sequences with different sky backgrounds were selected for testing. Figure 5 shows the target detection results of different algorithms. The red boxes in Figure 5 represent the targets detected under the optimal threshold of each algorithm.

As shown in Figure 5, it is indicated that for image sequence 1 and image sequence 2 , the bilateral filter and the proposed algorithm IDMST can successfully detect the target, but for the local entropy and max-mean method, the false alarm points are generated. Due to a lot of strong cloud clutters in image sequence 3, Only IDMST can detect the small target correctly, while other methods produce false alarm targets.

In conclusion, the IDMST can achieve good detection results in different backgrounds.

To evaluate the small target detection performance, the detection rate $\left(p_{d}\right)$ and the false alarm rate $\left(p_{f}\right)$ are used to measure. Both are defined as follows:

$$
\begin{aligned}
& p_{d}=\frac{N_{d}}{N_{t}}, \\
& p_{f}=\frac{N_{f}}{M},
\end{aligned}
$$

where $N_{d}$ is the number of correctly detected small targets, $N_{t}$ is the total number of real small targets, $N_{f}$ is the number of misdetected small targets, and $M$ is the total number of frames in the image sequence. Provided that the detection rate is higher and the false alarm rate is lower, the detection performance of the algorithm is better. 
TABLE 2: LSCR comparison results of different algorithms in different scenarios/units: $\mathrm{dB}$.

\begin{tabular}{lccc}
\hline Method & Original image 1 & Original image 2 & Original image 3 \\
\hline Bilateral filter & 9.4452 & 13.5572 & 14.5049 \\
Local entropy & 6.2632 & 6.2205 & 5.7345 \\
Max-mean & 7.5338 & 6.9543 & 7.7764 \\
IDSMT & 13.1539 & 18.3647 & 21.5173 \\
\hline
\end{tabular}

TABLE 3: SCRG comparison results of different algorithms in different scenarios/units: dB.

\begin{tabular}{lccc}
\hline Method & Original image 1 & Original image 2 & Original image 3 \\
\hline Bilateral filter & 1.5182 & 2.4428 & 2.6089 \\
Local entropy & 1.0067 & 1.1208 & 1.0314 \\
Max-mean & 1.211 & 1.2530 & 1.3987 \\
IDSMT & 2.1144 & 3.3091 & 3.8702 \\
\hline
\end{tabular}

TABLE 4: BSF comparison results of different algorithms in different scenarios.

\begin{tabular}{lccc}
\hline Method & Original image 1 & Original image 2 & Original image 3 \\
\hline Bilateral filter & 20.5727 & 5.7185 & 11.8676 \\
Local entropy & 0.2159 & 0.2136 & 0.1423 \\
Max-mean & 5.0018 & 2.01 & 4.0039 \\
IDSMT & 27.5659 & 9.978 & 16.118 \\
\hline
\end{tabular}

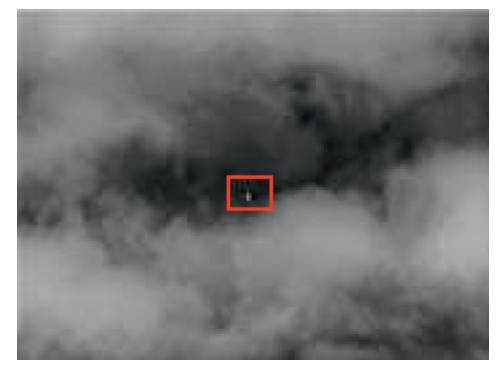

(a)

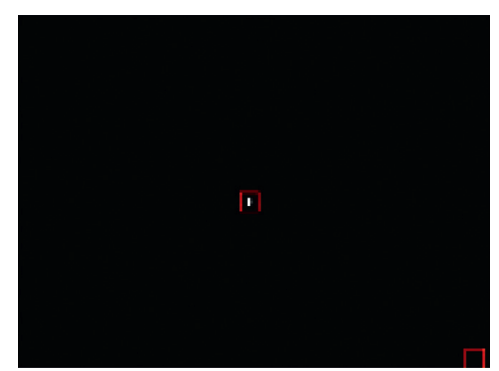

(d)

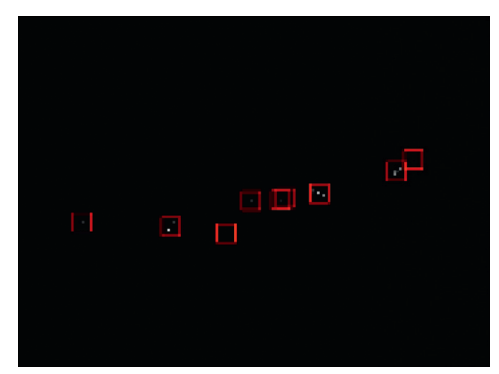

(g)

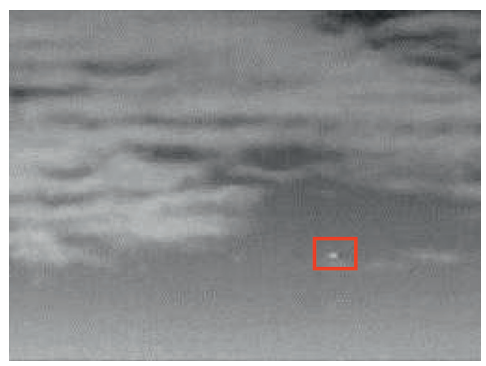

(b)

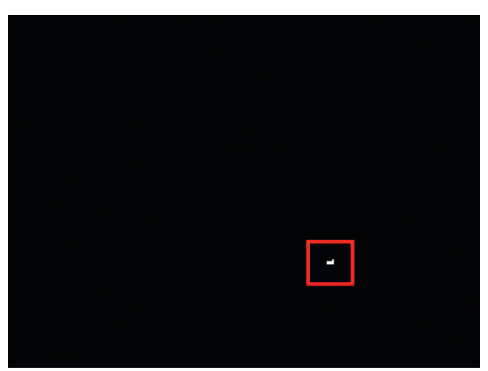

(e)

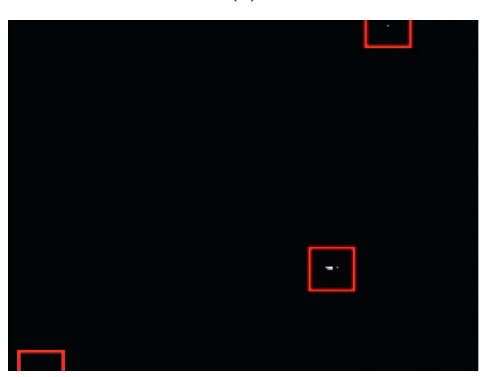

(h)

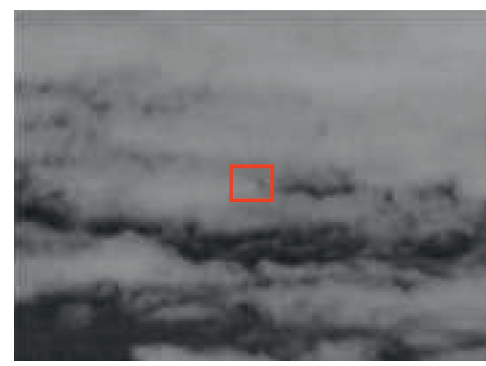

(c)

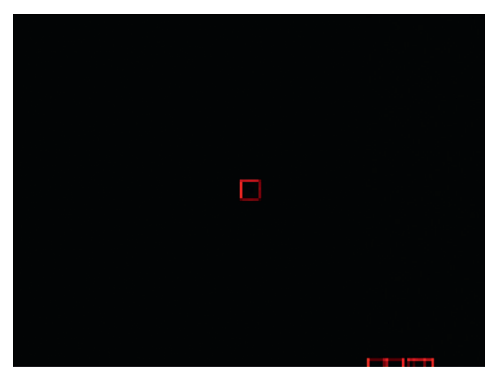

(f)

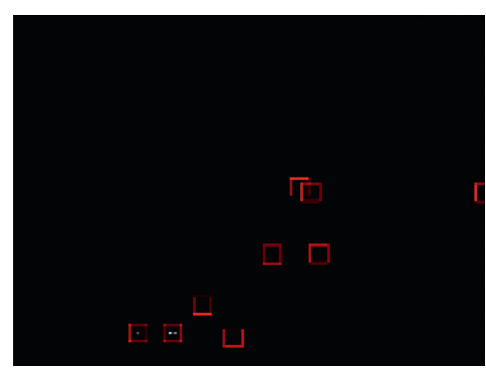

(i)

Figure 5: Continued. 


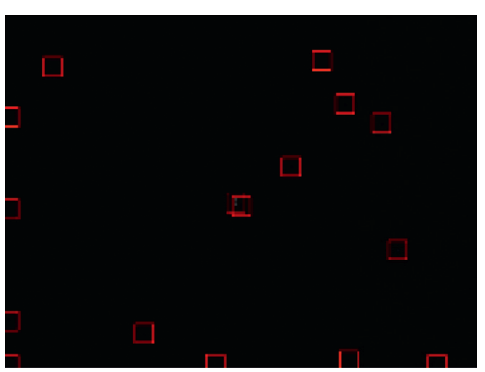

(j)

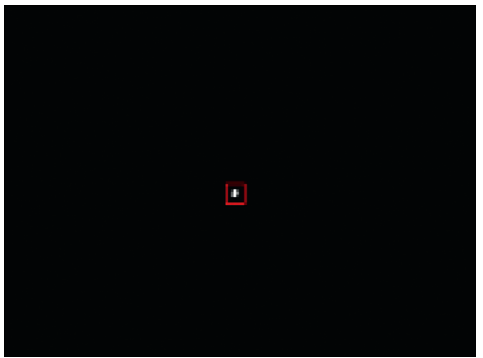

$(\mathrm{m})$

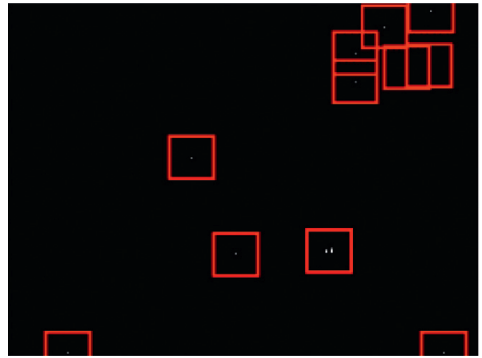

$(\mathrm{k})$

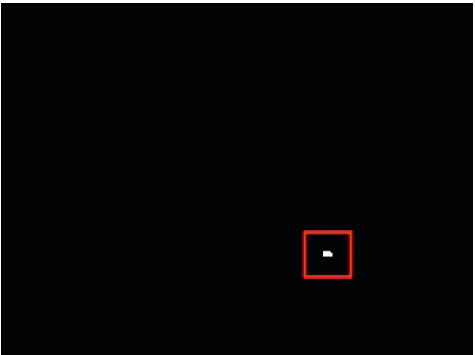

(n)

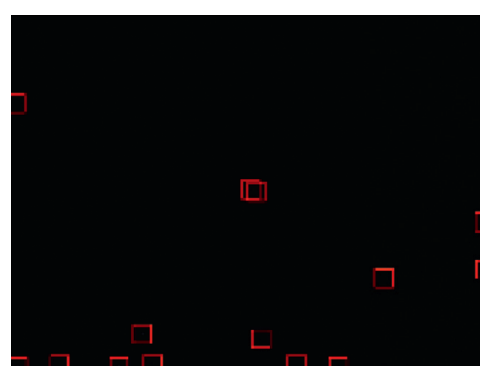

(1)

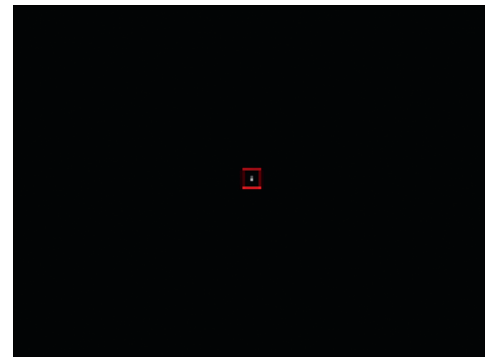

(o)

FIgURE 5: Test results of different algorithms. (a) Image sequence 1 included infrared original image 1. (b) Image sequence 2 included infrared original image 2. (c) Image sequence 3 included infrared original image 3. (d) Bilateral filter 1. (e) Bilateral filter 2. (f) Bilateral filter 3. (g) Local entropy 1. (h) Local entropy 2. (i) Local entropy 3. (j) Max-mean 1. (k) Max-mean 2. (l) Max-mean 3. (m) IDMST 1. (n) IDMST 2. (o) IDMST 3.

TABle 5: Detection rate and false alarm rate of each algorithm.

\begin{tabular}{lcccccc}
\hline & \multicolumn{2}{c}{$\begin{array}{c}\text { Original image } \\
\text { sequence 2 }\end{array}$} & \multicolumn{2}{c}{$\begin{array}{c}\text { Original image } \\
\text { sequence 2 }\end{array}$} & \multicolumn{2}{c}{$\begin{array}{c}\text { Original image } \\
\text { sequence 3 }\end{array}$} \\
& $p_{d}$ & $p_{f}$ & $p_{d}$ & $p_{f}$ & $p_{d}$ \\
\hline Bilateral filter & 1 & 0.11 & 1 & 0.03 & 0.99 & 0.14 \\
Local entropy & 0.93 & 1.46 & 1 & 0.16 & 0.72 \\
Max-mean & 0.98 & 1.52 & 0.8 & 0.63 & 0.96 \\
IDMST & 1 & 0.01 & 1 & 0 & 3.7 \\
\hline
\end{tabular}

By analyzing the detection rate and false alarm rate in Table 5, it can be concluded that the proposed algorithm can maintain high detection rate and low false alarm rate in three kinds of complex backgrounds. Therefore, the proposed algorithm IDMST has better detection performance.

\section{Conclusion}

To solve the problem that infrared small target detection is easy to be affected by clutter background, a method of infrared small target detection based on bilateral filtering and local entropy is proposed in this paper. The bilateral filtering is used to predict the image background, and then the dot product of the residual image and the local entropy image is calculated. The dot product result shows that the clutter background and the texture background are greatly suppressed while the small target is effectively retained. The guided filtering, which plays an important role in the separation of target and residual clutter, can enhance the target strength.
The experimental results show that the proposed algorithm owns better background suppression effect and superior target enhancement ability, as well as higher detection rate and lower false alarm rate, than other three methods. But in strong cloud clutter background, the false alarm rate is also introduced. The emphasis of future work is to further restrain the false alarm rate.

\section{Data Availability}

All data and models used during the study are available from the corresponding author upon request.

\section{Conflicts of Interest}

The authors declare that they have no conflicts of interest.

\section{Acknowledgments}

This work was supported by the National Natural Science Foundation of China under Grant no. 61861033 and the 
China Post-Doctoral Science Foundation under Grant no. 2017 M622108.

\section{References}

[1] D. Pang, T. Shan, W. Li, P. Ma, S. Liu, and R. Tao, "Infrared dim and small target detection based on greedy bilateral factorization in image sequences," Institute of Electrical and Electronics Engineers Journal of Selected Topics in Applied Earth Observations and Remote Sensing, vol. 13, no. 99, pp. 3394-3408, 2020.

[2] H. Ding and H. Zhao, "Adaptive method for the detection of infrared small target," Optical Engineering, vol. 54, no. 11, Article ID 113107, 2015.

[3] Y. Chen, B. Song, D. Wang, and L. Guo, "An effective infrared small target detection method based on the human visual attention," Infrared Physics \& Technology, vol. 95, pp. 128-135, 2018.

[4] R. G. Gavaskar and K. N. Chaudhury, "Fast adaptive bilateral filtering," Institute of Electrical and Electronics Engineers Transactions on Image Processing, vol. 28, no. 2, pp. 779-790, 2019.

[5] X. Bai, S. Qin, X. Du, and Y. Huang, "Improved adaptive bilateral filtering algorithm," Laser \& Optoelectronics Progress, vol. 57, no. 04, pp. 70-75, 2020.

[6] H. Zhu, X. Zhang, C. Xin, T. Hu, and P. Rao, "Dim small targets detection based on horizontal-vertical multi-scale grayscale difference weighted bilateral filtering," Journal of Infrared and Millimeter Waves, vol. 39, no. 4, pp. 513-522, 2020.

[7] J. Yuan and J. Wang, "Multi-scale moving target detection method based on improved bilateral filtering," Infrared Technology, vol. 41, no. 8, pp. 772-777, 2019.

[8] G. Wang, J. Tian, and J. Liu, "Infrared small objects detection based on local entropy method," Infrared and Laser Engineering, vol. 29, no. 4, pp. 26-29, 2000.

[9] G. Sun, S. Guo, and Z. Chen, "Real-time target detection algorithm of infrared imaging alarm system in panoramic field-of-view," Infrared and Laser Engineering, vol. 43, no. 7, pp. 2152-2158, 2014.

[10] H. Yang, H. Xia, X. Chen, S. Sun, and P. Rao, Application of image fusion in 3D reconstruction of space target," Infrared and Laser Engineering, vol. 47, no. 09, pp. 374-381, 2018.

[11] H. Deng, X. Sun, M. Liu, C. Ye, and X. Zhou, "Entropy-based window selection for detecting dim and small infrared targets," Pattern Recognition, vol. 61, pp. 66-77, 2017.

[12] H. Deng, X. Sun, M. Liu, C. Ye, and X. Zhou, "Infrared smalltarget detection using multiscale gray difference weighted image entropy," Institute of Electrical and Electronics Engineers Transactions on Aerospace and Electronic Systems, vol. 52, no. 1, pp. 60-72, 2016.

[13] X. Bai and Y. Bi, "Derivative entropy-based contrast measure for infrared small-target detection," Institute of Electrical and Electronics Engineers Transactions on Geoscience and Remote Sensing, vol. 56, no. 4, pp. 2452-2466, 2018.

[14] Z. Wang, J. Liu, and H. Deng, "Small-target infrared image processing based on novel weighted-local entropy," Journal of Huazhong University of Science \& Technology, vol. 45, no. 8, pp. 42-46, 2017.

[15] K. Qian, H. Zhou, H. Qin, S. Rong, D. Zhao, and J. Du, "Guided filter and convolutional network based tracking for infrared dim moving target," Infrared Physics \& Technology, vol. 85, pp. 431-442, 2017.
[16] G. Li, C. Long, C. L. P. Chen, and J. Zhou, "Integrating guided filter into fuzzy clustering for noisy image segmentation," Digital Signal Processing, vol. 83, pp. 235-248, 2018.

[17] C. Tomasi and R. Manduchi, "Bilateral filtering for gray and color images," in Proceedings of the Institute of Electrical and Electronics Engineers International Conference on Computer Vision, pp. 839-846, Bombay, India, January 1998.

[18] S. Ai, G. Zhang, and G. Lv, "A local entropy based algorithm for detecting IR target under sea background," Electronics Optics \& Control, vol. 15, no. 7, p. 75, 2008.

[19] S. D. Deshpande, M. H. Er, V. Ronda, and P. Chan, "MaxMean and Max-Median filters for detection of small targets," in Proceedings of the SPIE International Symposium on Optical Science, Engineering, and Instrumentation, vol. 3809, pp. 7483, Denver, CO, USA, July 1999.

[20] S. Zhang, X. Huang, and M. Wang, "Algorithm of infrared background suppression and small target detection," Journal of Image and Graphics, vol. 8, pp. 1039-1047, 2016.

[21] X. Shen, X. Cheng, and X. Wang, "Infrared dim-small object detection algorithm based on adaptive scale local contrast enhancement combined with visual attention mechanism," Infrared Technology, vol. 41, no. 8, pp. 764-771, 2019. 